\title{
A BULA DE MEDICAMENTOS: A IMPORTÂNCIA DA LEITURA DAS BULAS
}

\section{DRUG LABELING: THE IMPORTANCE OF READING THE DIRECTIONS FOR USE}

\author{
Gustavo de Cirqueira Rigotto ${ }^{1}$ \\ Regiane Rossi Oliveira Lima ${ }^{2}$ \\ Vera Lucia Mathias Geron ${ }^{3}$ \\ Jhonattas Munis de Souza ${ }^{4}$ \\ André Tomaz Terra Júnior ${ }^{5}$
}

\section{RESUMO}

A bula de medicamentos é um pequeno pedaço de papel que apresenta em seu conteúdo muita informação ao paciente, a pessoas leigas e profissionais da saúde. A leitura da bula deve ser considera como auxilio para o entendimento daquilo que se vai tomar, ou seja, do medicamento prescrito pelo médico. A falta ou a má leitura da bula pode dificultar o entendimento do processo que acontece durante o uso do medicamento ou até mesmo depois; por isso, os objetivos desse trabalho visa expandir o conceito de bula de medicamentos e descrever sobre a importância em fazer uma boa leitura para conseguir interpretar os seus dados. O estudo é uma revisão bibliográfica de materiais existentes sobre o tema em questão e tem o propósito de estender sobre o conhecimento científico já produzido sobre a bula de medicamentos, sua finalidade e a importância da leitura seja pelo próprio paciente ou por um farmacêutico. Contudo, considera-se ao fim deste estudo que diante de tantas reclamações sobre a bula de medicamentos no decorrer de vários anos e o fato da dificuldade que os pacientes encontram para fazer a leitura da bula e entender o que está escrito, levou a algumas mudanças e novas regras a serem definidas pela ANVISA - Agência Nacional de Vigilância.

Palavras-chave: Bula de medicamentos, Importância, Leitura.

\footnotetext{
${ }^{1}$ Discente do curso de Farmácia da Faculdade de Educação e Meio Ambiente (FAEMA), Ariquemes - RO.

${ }^{2}$ Graduada em Farmácia pela Faculdade de Educação e Meio Ambiente (FAEMA), Especialista em Farmácia Clínica; Coordenadora e docente da Faculdade de Educação e Meio Ambiente (FAEMA), Ariquemes - RO.

${ }^{3}$ Graduada em Ciências Biológicas, Graduada em Farmácia e Bioquímica. Especialista em Analises Clinica. Especialista em Manipulação Farmacêutica, Especialista em Didática do Ensino Superior e Mestre em Biologia dos Agentes Infecciosos e Parasitários pela Universidade Federal do Pará (UFPA). Coordenadora e docente da Faculdade de Educação e Meio Ambiente (FAEMA) Ariquemes - RO.
} 
${ }^{4}$ Engenheiro Químico pela Universidade Caxias dos Sul -UCS, Mestre em Engemharia de Processos e Tecnologia pela UCS, Docente da Faculdade de Educação e Meio Ambiente (FAEMA), Ariquemes - RO.

${ }^{5}$ Farmacêutico Industrial / Medicamentos, Mestre em Oncologia Clínica, Terapia Celular e Células Tronco pela Faculdade de Medicina de Ribeirão Preto - FMRP -USP, Docente do Curso de Graduação em Farmácia da FAEMA.

\title{
DRUG LABELING: THE IMPORTANCE OF READING THE DIRECTIONS FOR USE
}

\begin{abstract}
The labeling of medicines is a small piece of paper that shows in your content much information to the patient, lay people and health professionals. Reading the label it should be considered as an aid to understanding what is going to take, that is, the medicine prescribed by the doctor. The lack or poor reading the label may hinder the understanding of the process that takes place during the drug or even later; so the aim of this study aims to expand the concept of drug label and describe the importance of making a good read to be able to interpret your data. The study is a literature review of existing materials on the subject in question and aims to expand on the scientific knowledge produced on the label of medication, its purpose and the importance of reading either by the patient or by a pharmacist. However, it is considered the end of this study with so many complaints about the labeling of drugs over several years and the fact of the difficulty that patients are to make reading the label and understand what it says, has led to some changes and new rules to be defined by ANVISA National Health Surveillance Agency.
\end{abstract}

Keywords: Bull of drugs, importance, Reading.

\section{INTRODUÇÃO}

Bula é o nome que se dá ao papel que acompanha as embalagens dos medicamentos onde apresenta uma série de informações obrigatórias e fornecidas pelos laboratórios farmacêuticas dirigidas aos usuários ou aos profissionais da saúde ${ }^{(1)}$.

A Agência Nacional de Vigilância Sanitária - ANVISA é o órgão responsável em registrar as informações dos medicamentos sua regulação, análise e aprovação, sendo que aqui no Brasil, o conteúdo da bula de medicamentos também é submetido a este órgão ${ }^{(2)}$. 
Os medicamentos fabricados aqui no Brasil devem ter acrescentados em suas embalagens às bulas contendo informações. Essa legislação também compete até mesmo aos remédios importados (3)

Quando a pessoa que vai utilizar um medicamento e busca informações sobre ele, a primeira coisa que procura dentro da caixa é a bula, pois apresentam em forma escrita tais explicações. Os problemas que surgem para saúde devido à má administração do remédio pela pessoa doente, podem ser evitados se antes de tomar qualquer remédio à bula for lida ${ }^{(4)}$.

Todo paciente tem o direito em saber as informações que estão nas bulas de medicamentos em suas seções sobre o produto e outros conhecimentos legais, sendo que a Portaria da Secretaria de Vigilância Sanitária ${ }^{\circ} 110$, necessariamente regulamentou a obrigatoriedade em colocar dentro das embalagens dos medicamentos a bula com linguagem para o paciente ${ }^{(2)}$.

A leitura é essencial no cotidiano da vida de qualquer ser humano na busca por mais informações, estudos ou por atualizações. Ler uma bula de remédio, principalmente quanto este remédio será ingerido torna-se de grande relevância no entendimento da mesma. Quando o usuário necessita de uma noção mais segura sobre o seu medicamento, a bula relata essas informações ${ }^{(5)}$.

Toda bula consta mais detalhes do que aqueles que o médico informa no momento da consulta; sendo que a linguagem técnica não transmite nenhum conhecimento específico ao usuário, mas por ser um medicamento os fornecedores são obrigados a inserir tudo no contexto ${ }^{(6)}$.

A falta de leitura pode impedir a forma de interpretação de um texto e a bula de medicamentos está inserida neste contexto. Em meio a tantas informações existentes na bula é possível que a pessoa que vai ingerir o remédio sinta-se confuso e entenda de forma errada o que está escrito $^{(7)}$, por isso a necessidade em fazer com calma e detalhada leitura.

As informações que são apresentadas nas bulas são de fácil compreensão e muito transparente no que o paciente deve ou não fazer antes de tomar o remédio e quais os cuidados que devem ter se for bem lida ${ }^{(8)}$. O farmacêutico pode ser de grande ajuda no momento dessa leitura. Sem ter vergonha no momento da compra, o paciente pode e deve pedir auxílio quanto ao teor da bula, sua estrutura e compreensão.

No Brasil, as mudanças relacionadas à bula de medicamento começaram a ser revisada pela Agência Nacional de Vigilância Sanitária - ANVISA e restruturada por outros órgãos competentes, devido às reclamações que surgiam diante do texto, do tamanho da letra, da difícil linguagem, entre outros detalhes, propondo melhorias em relação às informações citadas ${ }^{(9)}$. 
O presente trabalho tem como objetivo expandir sobre a importância da bula de medicamentos, seus princípios, suas regras e sobre a nova forma que deve ser apresentada ao consumidor. Também tem como objetivo apresentar da leitura da mesma por pacientes leigos ou não, na hora da compra, ou antes, de tomar o medicamento.

Esta revisão bibliográfica está sendo realizada devido ao histórico de conceitos sobre a bula direcionada ao paciente, sobre a legislação na área de bulas de medicamentos e suas várias inovações direcionadas aos pacientes ou leigos.

Nota-se que ainda são necessárias algumas mudanças nas bulas que acompanham os medicamentos para que estas sejam de fácil entendimento e interpretação para o usuário, independentemente da escolaridade ou da classe social deste usuário, sendo que este entendimento acontecerá no momento em que se faz a leitura. Por isso a classificação de importante para a leitura da bula de medicamentos.

\section{MATERIAIS E MÉTODOS}

Para melhor compreensão e análise das informações, os referenciais científicos foram agrupados quanto a semelhança dos objetivos específicos. O delineamento do estudo foi de 2008 a 2015. A coleta do material foi realizada no período de setembro e outubro de 2015. Os critérios de inserção para revisão de literatura foram todos os periódicos disponíveis nas bases de dados nacionais e internacionais.

Entretanto os critérios de exclusão de revisão de literatura foram os periódicos que não estavam disponíveis completos e se encontravam sob a forma de resumo e não coerentes com as categorias propostas na pesquisa. Foram utilizados no total de 27 referências.

Realizaram-se buscas baseadas em uma revisão bibliográfica atual do tipo exploratória descritiva, utilizando fontes eletrônicas e plataformas científicas de pesquisa, como: Scientific Electronic Library Online - Scielo, Biblioteca Virtual em Saúde (BVS), Portal do Governo Federal, Conselho Federal de Farmácia - CFF, Revistas online, Google Acadêmico.

\section{REVISÃO E LITERATURA}

\subsection{BULA DE MEDICAMENTOS}


Quando é necessário identificar alguma doença ou mesmo evitar, buscar alívios para a dor e suas alterações ou até mesmo a cura, os medicamentos são os principais elementos indicados para tal função e são imprescindíveis neste processo, seguindo um critério de controles analisados e determinados pela ANVISA ${ }^{(10)}$.

As informações direcionadas como científicas, geralmente são consideradas como complicadas e de difícil interpretação. Um documento que acompanha os medicamentos definido como bula e considerado como legítimo, para ser lido e entendido mesmo com anotações técnicocientífica deve ser de fácil compreensão e com uma linguagem simplificada para os leigos ${ }^{(11)}$.

Os usuários de qualquer tipo de medicamento precisam saber da importância da bula como meio de comunicação, reconhecendo o quanto é fundamental para todos os tipos e suspeitas de riscos da automedicação, da forma como deve ser realizado o tratamento, como o remédio deve ser ingerido e principalmente receitado por algum profissional da saúde competente para tal ${ }^{(12) .}$

O que defini a bula como uma comprovação obtida do relato das pesquisas clínicas de natureza técnico-científica são as informações acerca do documento provindo dos resultados do desenvolvimento de um medicamento ${ }^{(13)}$.

\subsection{A IMPORTÂNCIA DA LEITURA DA BULA DE MEDICAMENTOS}

Os problemas com a leitura, compreensão do texto, tamanho da letra na maioria das vezes muito pequena, o exagero de informações em termos desconhecidos pelos pacientes, frases longas e complicadas e uso de normas em excesso apresenta-se como uma bula com problemas de interpretação ${ }^{(14)}$. A bula é dirigida a dois tipos de leitores, o profissional de saúde como os médicos, enfermeiros, farmacêuticos e o paciente, considerado como leigo.

Para os cidadãos com pouco estudo que sentem dificuldades no momento de uma leitura e de interpretar o que é lido, ou por um idoso, algumas bulas são consideradas difíceis de entender. Mas a escolaridade em grau maior não significa que o paciente compreenderá o conteúdo das bulas, sendo informação segundo o Índice de Letramento Científico ${ }^{(15)}$.

No Brasil, de 1946 até 2009, foram divulgados dispositivos legislativos reeditados pela ANVISA sobre o desenvolvimento das normas que regulamentam a bula de medicamentos, sendo que os nove dispositivos eram relacionados aos obstáculos referentes ao entendimento das bulas por pessoas que usam aquele tipo de remédio ou simplesmente por pessoas que leem a bula ${ }^{(16) .}$ 
A Agência Nacional de Vigilância Sanitária (ANVISA), através da Resolução da Diretoria Colegiada $n^{\circ} 47$ de 2009, estabeleceu regras para as bulas de medicamentos regulamentando alguns itens como: tamanho da fonte, conhecimento sobre os remédios genéricos e equivalentes, acesso às recomendações da bula para os deficientes visuais e precisão para os usuários e capacitados na área de saúde, além da disponibilidade eletrônica por mais conhecimentos e com mais facilidade ${ }^{(17)}$.

Aqui no Brasil quem utiliza o sistema de saúde tem o direito garantido pela Lei Orgânica da Saúde às informações da bula. Mas todo esse conhecimento do conteúdo disponibilizado e da noção de acesso e direito apenas contribuirá positivamente através da clareza da leitura adequada às precisões da informação e o nível de conhecimento das pessoas que utilizam o sistema ${ }^{(13)}$.

Ler e interpretar um texto é essencial para o entendimento; e com a bula não seria diferente já que ela traz relatos sobre a apresentação técnica do medicamento, noções necessárias ao usuário e situações legais competentes. Toda embalagem de medicamento obrigatoriamente deve conter uma bula ${ }^{(18)}$.

Ainda para facilitar leitura da bula de medicamentos, assim como a mudança do tamanho da letra houve a mudança no espaçamento que separa uma linha da outra entre os parágrafos ${ }^{(19)}$.

\subsection{LINGUAGENS TÉCNICA DA BULA}

A leitura de uma bula algumas vezes é complexa e pode deixar o paciente alarmado devido às informações que o laboratório fabricante relata sobre as reações que o medicamento pode provocar. No entanto, mesmo tendo uma resistência adversa ao vocabulário técnico, o usuário não deve deixar de ler a bula ${ }^{(20)}$.

Todas as informações referentes ao medicamento são de responsabilidade do "fabricante,

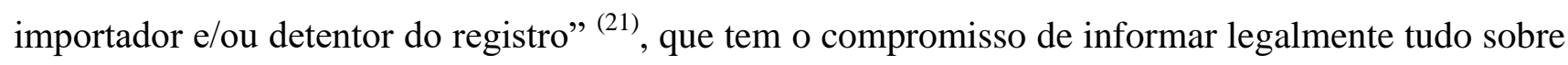
o produto, a forma eficiente de usa-lo aos pacientes e pessoas envolvidas na área da saúde ${ }^{(21)}$.

A particularidade das bulas é primordial no uso adequado e seguro dos medicamentos após a receita ministrada e também após a venda nas farmácias. Todo o esclarecimento sobre o medicamento e a forma com usar está disponível na bula de uma forma definida ${ }^{(22)}$.

Quando o cidadão necessita de informações geralmente procura algo para ler. Neste caso com os pacientes a situação não é diferente, pois irá pesquisar nas bulas e em outros materiais recomendações sobre aquilo que vai ingerir, mas infelizmente esses materiais têm um linguajar difícil 
de ser entendido, principalmente aos que não têm estudo suficiente para entender o que está escrito em um grau de linguagem muito complexa para a grande parte dos consumidores, complicando a leitura ${ }^{(12)}$.

As informações da bula são fundamentais para a orientação do paciente. A exclusão de dados nos tópicos relacionados na bula como as contraindicações, a ação do medicamento ou o seu efeito ao organismo, leva o usuário as riscos existentes e de relevância nas intoxicações e outros problemas durante o tratamento ${ }^{(23)}$.

Diante de tal circunstância, a ANVISA relata que as mudanças das novas bulas de medicamentos para os pacientes estarão em forma de perguntas e respostas ${ }^{(10)}$, seguindo a ordem:

- Como este medicamento funciona?

- Por que este medicamento foi indicado?

- Quando não devo usar este medicamento?

- Como devo usar este medicamento?

- Quais os males que este medicamento pode causar?

- O que fazer se alguém usar uma grande quantidade deste medicamento de uma só vez?

- Onde e como devo guardar este medicamento?

A essência da comunicação determinada nas bulas de medicamentos é de grande importância ao usuário. As questões que devem ser claramente dirigidas aos pacientes relacionam-se ao conteúdo estar facilmente disponível e em linguagem que possam ser lidas e compreendidas ${ }^{(3)}$.

\subsection{O PAPEL SOCIAL DO FARMACÊUTICO DIANTE DA LEITURA DAS BULAS}

O Conselho Federal de Farmácias (CFF) atribui aos farmacêuticos várias funções desconhecidas pelo o público em geral, podem trabalhar em 71 (setenta e uma) área diferentes. Os farmacêuticos são os responsáveis em relatar informações sobre o medicamento receitado e sanar dúvidas do usuário ${ }^{(24)}$.

O farmacêutico pode estar apto a realizar exames clínicos, trabalhar em indústrias alimentícias, se envolver na pesquisa e elaboração de novos medicamentos, além de se envolver socialmente no primeiro contato como o paciente alertando sobre o uso do remédio e as informações necessárias na bula ${ }^{(25)}$. 
O farmacêutico tem grande importância no diálogo, conhecimento e comunicação junto ao doente em relação aos remédios que usará e também é o guia para que a pessoa entenda a enfermidade em que se encontra tirando dúvidas e sendo orientado pelo profissional ${ }^{(26)}$.

Devido ao seu conceito, as mudanças realizadas nas bulas dos remédios são significativas tanto para o profissional da saúde quanto para o usuário. Todas as informações sobre como tomar o remédio, o horário e possíveis complicações são consideradas primordiais pelo farmacêutico ${ }^{(27)}$.

\section{CONSIDERAÇÕES FINAIS}

A bula é considerada como um instrumento educativo que auxilia o entendimento e compreensão daquilo que vai ser usado.

Percebe-se que as quantidades de informações existentes nas bulas de medicamentos são importantes para o paciente, usuário contínuo, profissionais da saúde e farmacêuticos. Essas informações indicam todo o processo de como usar, do que fazer ou não fazer enquanto estiver tomando o medicamento ou aplicando em alguma parte do corpo, das reações que podem ou não causar ao organismo.

Diante de todas as circunstâncias apresentadas em relação à importância da bula de medicamentos, nota-se que a única forma de reconhecer esses itens e compreender o conteúdo existente é através da leitura. Mas somente a leitura não ajudará em questão, também deve haver o entendimento do que está sendo lido, ou seja, a interpretação correta dos dados informados.

Verifica-se que a leitura será bem realizada pelo paciente se o texto possuir elementos e condições de compreensão de acordo com o nível de conhecimento do leitor; a linguagem técnicacientífica dirigida apenas para profissionais da saúde dificultam a interpretação do leigo, assim como o tamanho da fonte também prejudica a leitura.

Em relação à linguagem técnica apresentada na bula, acrescenta-se certo receio ao usuário caso ele não consiga entender o que está escrito. Este receio ou desconhecimento da maneira correta de como usar o remédio poderá prejudicar ou modificar o tratamento prescrito pelo médico, acarretando assim em possíveis complicações ou não conseguindo o efeito desejado.

Compreende-se que essa linguagem técnica poderá desmotivar o paciente a usar o medicamente, principalmente quando se refere às complicações que o uso pode acarretar. tamanho da letra existente nas bulas, o espaçamento entre as linhas, as divisões em forma de colunas, a linguagem de difícil compreensão foram alguns dos itens de reclamações apresentadas aos órgãos 
competentes sobre o assunto. Nas várias mudanças que foram realizadas nas Resoluções da Diretoria Colegiada da Agência Nacional de Vigilância Sanitária - ANVISA, a questão foi em solucionar este problema que prejudicava muitos usuários.

Faz-se entender que quando o usuário depara-se com algum obstáculo diante da leitura, é seu direito procurar ajuda, tornando neste sentido dever do farmacêutico em ajudar no processo de entendimento e interpretação da bula para que não aconteça nenhum erro prejudicial à saúde.

Conclui-se então, que a leitura é um meio fundamental para todo o conhecimento e informações necessárias diante de uma situação seja ela referente á saúde ou não. Mas em relação à saúde e ao bem estar do cidadão, a bula de medicamentos é considerada como o meio mais prático e próximo do paciente, sendo que o conteúdo apresentado ao leitor seja considerado fácil e nítido, transmitindo neste sentido uma segurança em ingerir ou usa o remédio prescrito.

Nos resultados obtidos através da pesquisa percebe-se que os pacientes têm conhecimento da bula que acompanha a caixa de remédios, mas muitos a ignoram, pois consideram a leitura difícil diante da linguagem técnica ou devido ao tamanho da letra e sua estrutura e diante dessas situações necessitam da ajuda do farmacêutico ou de outros.

\section{REFERÊNCIAS}

1 - Kamisaki, M. S., et al. Bulas e Cartelas de Medicamentos: Possíveis soluções de leiturabilidade através do Design Gráfico. Arcos Design, vol.6, no 1, dezembro 2011. [citado em 1 de outubro 2015]. Disponível em: < http://www.e-publicacoes.uerj.br/index.php/arcosdesign/issue/view/948/showToc>

2 - BRASIL, 2011. ANVISA. Agência Nacional de Vigilância Sanitária. Regulamentação de Bulas de Medicamentos no Brasil - RDC n 47/2009, janeiro de 2011. [citado em 1de outubro de 2015]. Disponível em: < http://www.anvisa.gov.br/medicamentos/bulas/rdc_47.pdf

3- Souza J. P. R. de, et al. O paciente e a bula e suas maiores dificuldades. Revista Faculdade Montes Belos, v. 7, $\mathrm{n}^{\mathrm{o}}$ 2, 2014, p. 10-22. [citado em 5 de outubro 2015]. Disponível em:< http://www.fmb.edu.br/revistaFmb/index.php/fmb/article/view/117/112>

4 - Horne, F. Sabia que é muito importante ler a bula antes de usar um medicamento? Publicado em: 04 de março de 2015. [citado em 3 de outubro de 2015]. Disponível em: < http://www.reclameaqui.com.br/noticias/noticias/sabia-que-e-muito-importante-ler-a-bula-antes-deusar-um-med_1218/>

5 - DIAS, P. Embalagem, rótulo e bula de medicamentos. [citado em 3 de outubro de 2015]. Disponível em: < http://cadernodefarmacia.blogspot.com.br/2013/03/embalagem-rotulo-e-bula-dosmedicamentos.html $>$ 
6 - ABC. MED. BR, 2014. Relação médico-paciente para pacientes. [citado em 12 outubro de 2015]. Disponível em: $<$ http://www.abc.med.br/pvidasaudavel/574947/relacao+medico+paciente+para+pacientes.htm>

7 - Como ler uma bula de medicamentos. [citado em 4 de outubro de 2015]. Disponível em: < http://www.posologiademedicamento.com.br/como-ler-uma-bula-de-medicamento/>

8 - Porque ler as bulas é importante. 28/05/2015. [citado em 29 de setembro 2015]. Disponível em: < http://www.bulamedicamento.com.br/porque-ler-as-bulas-e-importante/>

9 - Brasil. ANVISA - Agência Nacional de Vigilância Sanitária. Bulas. Histórico. [citado em 30 de setembro de 2015]. Disponível em: http://portal.anvisa.gov.br/wps/content/Anvisa+Portal/Anvisa/Profissional+de+Saude/Assunto+de+In teresse/Bulas+e+Rotulos+de+Medicamentos/Bulas/B+Historico+-+Bulas>

10 - Brasil. 2010. ANVISA. O que devemos saber sobre medicamentos. [citado em 29 de setembro de 2015]. Disponível em:< http://portal.anvisa.gov.br/wps/wcm/connect/92aa8c00474586ea9089d43fbc4c6735/Cartilha\%2BBA IXA\%2Brevis\%C3\%A3o\%2B24_08.pdf?MOD=AJPERES>

11- Pires, L. D. Relação que o usuário tem com a bula do medicamento. ICTQ - Instituto de Ciência Tecnologia e Qualidade, 2014. [citado em 30 de setembro de 2015]. Disponível em:< http://ictq.com.br/portal/colunas-materias/relacao-que-o-usuario-tem-com-a-bula-do-medicamento>

12 - Volpato, L. F. Bulas de medicamentos e profissionais de saúde: ajudam ou complicam a compreensão dos usuários? Revista de Ciências Farmacêutica Básica e Aplicada, 2009; 30(3): 309314. [citado em 30 de setembro de 2015]. Disponível em: <http://servbib.fcfar.unesp.br/seer/index.php/Cien_Farm/article/viewFile/623/887>

13 - Fujita, P. L. et al., A bula de medicamentos e a regulação de suas configurações em termos de forma e conteúdo no Brasil. [citado em 29 de setembro de 2015]. Disponível em: < http://www.scielo.br/pdf/sausoc/v23n1/en_0104-1290-sausoc-23-01-00277.pdf>

14 - Pires, L. D. A importância da bula. [citado em 30 de setembro de 2015]. Disponível em: $<$ http://www.guiadafarmacia.com.br/259-jun-14-sentir-dor-e-sinal-de-alerta/8074-a-importancia-dabula>

15- Vieira, V. Brasileiro sofre para interpretar até bula. O Estado de São Paulo. Data 16/06/2014. [citado em 1 de outubro de 2015]. Disponível em:

<http://www.educacao.estadao.com.br/.../geral.brasileiro-sofre-para-interpretar-ate-bula,...>.

16 - BRASIL, 2014. CFF. Conselho Federal de Farmácia. Código de Ética. Resolução nº 596, 21 de fevereiro 2014. [citado em 30 de setembro de 2015]. Disponível em: <http://www.cff.org.br/pagina.php?id=167>

17 - Almeida, S. M. A importância de ler a bula de medicamento. [citado em 3 de outubro de 2015]. Disponível em: <http://www.einstein.br/blog/paginas/post. aspx?post=1506> 
18 - Vieira, T. et al. Você lê a bula dos medicamentos que compra? Unimed, 2 de julho 2007. [citado

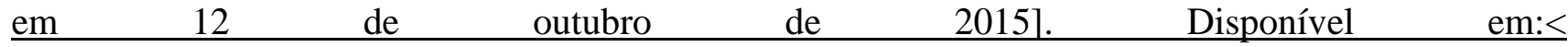
http://www.unimed.coop.br/pct/index.jsp?cd_canal=49146\&cd_secao=54398\&cd_materia=286945

19 - Aragão, B. Bula fácil de entender. Rede HumanizaSUS - Rede de colaboração para a humanização da gestão e da atenção no SUS, 2009. [citado em 12 de outubro de 2015]. Disponível em: $\langle$ http://www.redehumanizasus.net/4652-bula-facil-de-entender $>$

20 - Hospital Ministro Costa Cavalcanti. É medicamento? Leia a bula. Newsletter Edição 4, 2014. [citado em 2 de outubro de 2015]. Disponível em: $<$ http://www.hmcc.com.br/saibamais.php?artigo $=36>$

21 - Gonçalves, S. de A. Análise das bulas de medicamentos e percepção da sua importância pelos idosos. Universidade Católica de Brasília, 2013. [citado em 30 de setembro de 2015]. Disponível em: $<\quad$ http://www.bdtd.ucb.br/tede/tde_arquivos/17/TDE-2014-04-15T075444Z1760/Publico/Silmara\%20de\%20Almeida\%20Goncalves.pdf $>$

22 - Pires, C. et al. Legibilidade das Bulas de Medicamentos: Revisão sistemática. Revista de Saúde Pública, 2015; 49:4. [citado em 1 de outubro de 2015]. Disponível em: 〈http://www.revistas.usp.br/rsp/article/view/101889>

23 - Balbanil, A. P. S. et al. Bula de medicamentos para tratamento de rinites. Revista Brasileira de alergia e imunopatologia, 2003; 26(1):17-24. [citado em 30 de setembro de 2015]. Disponível em: <http://www.asbai.org.br/revistas/Vol261/bulas.htm>

24 - As mil e uma atividades do farmacêutico. Pfizer Institucional. [citado em 4 de outubro de 2015]. Disponível em: $<$ http://www.pfizer.com.br/noticias/mil-e-uma-atividades-do-farmac\%C3\%AAutico $>$

25 - Rocha, G. O papel social do farmacêutico. Ministério da Saúde. Blog da Saúde, 2014. [citado em 12 de outubro de 2015]. Disponível em: <http://www.blog.saude.gov.br/34398-o-papel-social-dofarmaceutico>

26 - Silva, V. da S. et al. O papel social do farmacêutico comunitário no aconselhamento ao paciente. Conselho Federal de Farmácia - CFF. Centro Brasileiro de Informação sobre Medicamentos. Boletim Farmacoterapêutica. Ano XIII. Números 4 e 5. jul-out/2008. [citado em 30 de setembro de 2015]. Disponível em: <http://www.cff.org.br/sistemas/geral/revista/pdf/67/057a064_farmacoterapeutica.pdf>

27 - Silva, A. M. Por uma bula mais clara. Conselho Federal de Farmácia - CFF. Guia da Farmácia, 2013. [citado em 1 de outubro de 2015]. Disponível em:< http://www.cff.org.br/noticia.php?id=1456> 